\title{
Medical and Dental Visits of Chronic Kidney Disease- Diagnosed Participants Analyzed From the Specific Health Checkups Results in Japan: TAMA MED Project-CKD
}

\author{
Tomohiro Kaneko a, f, Eitaro Kodani ${ }^{\text {, }}$, Hitomi Fujiic, Hiroyuki Nakamura ${ }^{\text {d }}$, \\ Hajime Sasabe ${ }^{d}$, Yutaka Tamura ${ }^{d}$, Shuichi Tsuruoka ${ }^{\mathrm{e}}$
}

\begin{abstract}
Background: Since 2012, Tama City has promoted the early detection of chronic kidney disease (CKD), through an initiative that measures serum creatinine as part of the specific health checkups. We examined preventive measures against CKD deterioration based on the outcomes of this initiative.
\end{abstract}

Methods: The complications, medication status, body mass index, smoking status and other determining factors were surveyed among CKD-diagnosed participants over 3 years between 2013 and 2015. Moreover, factors aggravating CKD were investigated via a survey of medical and dental visits based on health insurance claim data over the same period.

Results: There was an increased rate of comorbid hypertension with each increase in the CKD stage. Comorbidity rates of diabetes mellitus, dyslipidemia, obesity, and smoking increased until CKD stage G4, and then decreased from stage G5. A substantial number of participants with CKD stage G3b and above were not medicated despite comorbidities like hypertension, diabetes mellitus and dyslipidemia. While the rate of regular visits at medical institutions was seen to increase significantly in accordance with the worsening degree of CKD, there were also individuals who, despite having severe CKD, did not visit medical institutions specializing in internal medicine. The rate of dental visits decreased as the CKD stage increased, and further decreased as the diabetic control status worsened.

Conclusions: CKD patients should become aware of the importance

Manuscript submitted January 8, 2020, accepted January 17, 2020

aDepartment of Nephrology, Nippon Medical School Tama-Nagayama Hospital, Tokyo, Japan

${ }^{b}$ Department of Internal Medicine and Cardiology, Nippon Medical School Tama-Nagayama Hospital, Tokyo, Japan

'Tama-Center Mirai Clinic, Tokyo, Japan

dTAMA CITY Medical Association, Tokyo, Japan

eDepartment of Nephrology, Nippon Medical School, Tokyo, Japan

${ }^{f}$ Corresponding Author: Tomohiro Kaneko, Department of Nephrology, Nippon Medical School Tama-Nagayama Hospital, 1-7-1 Nagayama, Tama, Tokyo 206-8512, Japan. Email: tomohiro@nms.ac.jp

doi: https://doi.org/10.14740/jocmr4085 of the dental visit because only a limited number of patients with advanced $\mathrm{CKD}$ received dental care.

Keywords: Chronic kidney disease; Specific health checkups; CKD without proteinuria; Medical and dental visits

\section{Introduction}

Chronic kidney disease (CKD) is a precursor to end-stage renal disease that is on the rise worldwide and steadily increasing in Japan. Awareness-raising campaigns aimed at citizens and cooperation between primary care doctors and specialists have been sought as countermeasures $[1,2]$. In many cases CKD symptoms do not appear until the disease has progressed, so it is critical to prevent disease progression through the earliest possible detection and appropriate medical intervention.

In 2008, specific health checkups (Tokutei Kenshin) were initiated in Japan to detect lifestyle-related diseases in persons covered by the national health insurance. Although specific health checkups are useful in the diagnosis of metabolic syndrome [3], they are ineffective in screening for CKD because it is not mandatory to examine serum creatinine. However, in the Tama City suburbs of Tokyo, serum creatinine has been measured for all people who receive specific health checkups since 2012 (TAMA MED Project-CKD).

Based on this data, the current research investigated complicating symptoms, medication status, body mass index (BMI), smoking status, and various other factors in CKD-diagnosed participants. Moreover, using data of health insurance claims, the status of CKD-diagnosed participants' medical and dental visits was also investigated. Such an evaluation would arguably reveal the aggravating factors in CKD and aid in preventing its deterioration.

\section{Materials and Methods}

\section{Study design of the TAMA MED Project-CKD}

The TAMA MED Project-CKD was conducted as a retrospec- 
tive cohort study to examine the prevalence and incidence of $\mathrm{CKD}$ in a general population in conjunction with Project-AF and Frail which was initiated to clarify the prevalence and incidence of atrial fibrillation and frail $[4,5]$. The study protocol conformed to the Declaration of Helsinki and was approved by the ethics committee of our institution (approval number 529, 2017).

Participants with national health insurance who had undergone annual specific health checkups at a clinic or hospital belonging to the TAMA CITY Medical Association were sampled serially. All participants were aged 40 - 74 years upon admission as only individuals between these ages were eligible for specific health checkups on this insurance. All participants answered the questionnaire including measures on past history of cardiac disease and stroke, present illness, habitual status including current smoking, and medications. In addition, body height and weight, BMI, waist circumference, and systolic and diastolic blood pressure (BP) were measured in participants. This study also examined fasting plasma glucose (FPG) or hemoglobin Alc (HbA1c), serum high-density lipoprotein cholesterol (HDL-C), low-density lipoprotein cholesterol (LDL-C), triglycerides, aspartate transaminase (AST), alanine transaminase (ALT), $\gamma$-glutamyl transpeptidase $(\gamma$-GTP), and urine sugar and protein (using the test paper method) as essentials in line with the ordinance of the Ministry of Health, Labour and Welfare that referred to Article 157 in 2007. In addition, since 2008, a standard 12-lead electrocardiogram (ECG) has been recorded in all participants as a Tama City-specific mandatory optional examination [4], while serum creatinine has been measured since 2012. Estimated glomerular filtration rate (eGFR) was calculated using the following equation: for men, eGFR $\left(\mathrm{mL} / \mathrm{min} / 1.73 \mathrm{~m}^{2}\right)=194 \times($ age $)-0.287 \times($ serum Cre $(\mathrm{mg} / \mathrm{dL}))-1.094$, and for women, eGFR $(\mathrm{mL} / \mathrm{min} / 1.73$ $\left.\mathrm{m}^{2}\right)=194 \times($ age $)-0.287 \times($ serum $\mathrm{Cr}(\mathrm{mg} / \mathrm{dL}))-1.094 \times$ 0.739 [6]. Data from the period of $2013-2015$ were analyzed.

\section{Categories of degree of CKD worsening}

Level of renal dysfunction (G1 - G5) was determined based on eGFR measurements. Test strips were used to measure protein in urine and classified as normal for (-) or $( \pm)$, low levels for $(+)$ and high levels for $(++)$ or higher. CKD was defined as having proteinuria and/or eGFR less than $60 \mathrm{~mL} / \mathrm{min} / 1.73 \mathrm{~m}^{2}$. CKD categories were defined as follows based on heat map data [7]: 1) Low risk or no CKD (green): G1, 2 with urine protein levels of $(-)$ or $( \pm)$; 2) Moderately increased risk (yellow): G1, 2 with urine protein levels of $(+)$, or G3a with urine protein levels of $(-)$ or $( \pm)$; 3) High risk (orange): G1, 2 with urine protein levels of $(++)$ or more, G3a with urine protein levels of $(+)$, or G3b with urine protein levels of $(-)$ or $( \pm)$; and 4) Very high risk (red): G3a with urine protein levels $(++)$ or higher, $\mathrm{G} 3 \mathrm{~b}$ with protein levels of $(+)$ or higher, or G4, 5 .

\section{Definitions of other comorbidities}

Hypertension was defined as having a systolic BP $\geq 140 \mathrm{~mm}$
$\mathrm{Hg}$ and/or receiving antihypertensive medication. Diabetes mellitus was defined as having an FPG $\geq 126 \mathrm{mg} / \mathrm{dL}$ or $\mathrm{HbA} 1 \mathrm{c}$ $\geq 6.5 \%$ (National Glycohemoglobin Standardization Program, NGSP), and/or receiving medical treatment with oral hypoglycemic agents and/or insulin. Hyperlipidemia was defined as having an LDL-C $\geq 140 \mathrm{mg} / \mathrm{dL}$ and/or receiving antihyperlipidemic medication.

\section{Survey of medical and dental visits}

Medical and dental visits of CKD-diagnosed participants were investigated using data of health insurance claims linked to medical checkups of patients. Specific health checkups in Japan include a medical doctor's consultation, but in this study, this consultation was distinguished from "medical visits". We calculated for each category of CKD group whether they were visiting a medical institution and dental clinic or not.

We also investigated whether medical institutions advocate the ability to treat internal diseases (specializing in internal medicine). Regular visit was defined as the same medical institution is regularly visited within 4 months and has been continuing for more than 1 year. This was defined by the fact that doctors cannot give more than 3 months of medicine in Japanese medical facilities.

\section{Statistical analysis}

Data are presented as mean \pm standard deviation (SD). The statistical significance of differences between groups was analyzed using Fisher's exact test. $\mathrm{P}$ values $<0.05$ were considered as statistically significant in all analyses.

\section{Results}

\section{Prevalence of CKD in 2015}

The background of participants who underwent specific health checkups in 2015 are detailed in Table 1. As of December 1, 2015 , the total population of Tama City was 147,873 , with 72,895 individuals aged between 40 - 74 years (48\% men). The number of national health insurance subscribers in this age group was $37.2 \%$, and the rate of receiving specific health checkups was $45.8 \%$ in 2015 . Therefore, the total number of 12,303 people in this study was $16.9 \%$ of this age group in Tama City. Patients already taking medication for hypertension or diabetes mellitus numbered $34.2 \%$ and $8.2 \%$ of the total sample respectively. Table 2 outlines the urine protein levels of individuals who underwent checkups classified according to GFR stage. Although urine test results were unavailable for 29 individuals, according to the health insurance claim data these individuals were 14 dialysis patients with CKD G5. There were 2,437 CKD-diagnosed participants, accounting for $19.8 \%$ of the total. Of the total CKD population, $77.8 \%$ had eGFR less than $60 \mathrm{~mL} / \mathrm{min} / 1.73 \mathrm{~m}^{2}$ without proteinuria. 
Table 1. Characteristics of Participants

\begin{tabular}{|c|c|}
\hline Number of subjects & 12,303 \\
\hline Age (years) & $67.0 \pm 7.3$ \\
\hline Sex: men, women (\%) & 5,034 (40.9), 7,269 (59.1) \\
\hline BMI $\left(\mathrm{kg} / \mathrm{m}^{2}\right)$ & $22.6 \pm 3.3$ \\
\hline Systolic BP (mm Hg) & $127.2 \pm 16.5$ \\
\hline Diastolic BP (mm Hg) & $74.5 \pm 10.4$ \\
\hline Current smoker & $2,697(22.2)$ \\
\hline \multicolumn{2}{|l|}{ Comorbidities } \\
\hline Cardiac disease ${ }^{\mathrm{a}}$ & $749(6.1)$ \\
\hline Hypertension & $5,235(42.6)$ \\
\hline Diabetes mellitus & $1,603(13.0)$ \\
\hline Stroke/TIA & $488(4.0)$ \\
\hline Metabolic syndrome & $1,837(14.9)$ \\
\hline \multicolumn{2}{|l|}{ Blood examinations } \\
\hline HDL-C (mg/dL) & $64.5 \pm 16.6$ \\
\hline LDL-C (mg/dL) & $124.6 \pm 30.4$ \\
\hline Triglycerides (mg/dL) & $114.4 \pm 80.1$ \\
\hline HbA1c (NGSP) $)^{b}(\%)$ & $5.7 \pm 0.6$ \\
\hline \multicolumn{2}{|l|}{ Urinalysis } \\
\hline Urine sugar & $280(2.3)$ \\
\hline Urine protein & $517(4.2)$ \\
\hline \multicolumn{2}{|l|}{ EKG } \\
\hline $\mathrm{AF}$ & $175(1.4)$ \\
\hline \multicolumn{2}{|l|}{ Medications } \\
\hline For hypertension & $4,206(34.2)$ \\
\hline For diabetes mellitus & $1,011(8.2)$ \\
\hline For dyslipidemia & $2,693(21.9)$ \\
\hline
\end{tabular}

Data are given as $n(\%)$ or mean \pm SD. BMI: body mass index; $B P$ : blood pressure; TIA: transient ischemic attack; HDL-C: high-density lipoprotein cholesterol; LDL-C: low-density lipoprotein cholesterol; NGSP: National Glycohemoglobin Standardization Program; JDS: Japan Diabetes Society; AF: atrial fibrillation; EKG: electrocardiogram. ${ }^{a}$ Cardiac disease was self-reported. ${ }^{\text {b }} \mathrm{HbA} 1 \mathrm{c}$ (JDS) $+0.4 \%$.

\section{Lifestyle disease status according to CKD stage}

Lifestyle disease comorbidity and medication status of par- ticipants who underwent specific health checkups in 2015 are presented below (Table 3). Hypertension comorbidity rates rose with increments in CKD stages. Diabetes mellitus, dyslipidemia, and obesity comorbidity rates were seen to increase until CKD stage G4, before decreasing from stage G5. There were 14 individuals receiving dialysis within CKD stage G5, and the number of patients with a BMI $<18.5$ increased rapidly. There was a substantial number of participants $\geq$ CKD stage G3b who were not on medication despite having comorbidities with hypertension, diabetes mellitus, or dyslipidemia. Smoking comorbidity rates for CKD stage G3b and G4 patients were high.

\section{The rates of medical and dental visits of CKD-diagnosed participants}

Figure 1 presents the regular visits at medical institutions of participants undergoing health checkups over 3 years from 2013, excluding participants without urine test results. This was presented according to the CKD risk classifications, not including 14 dialysis patients in the very high risk group. The rates of regular visits at medical institutions increased as CKD risk increased; even when limited to medical institutions specializing in internal medicine, over $80 \%$ of health checkup recipients in the high risk group visited regularly. Figure 2 depicts the rates of dental visits of CKD-diagnosed participants compared to regular visits at medical institutions specializing in internal medicine over 3 years from 2013. In 2014 and 2015, the very high risk group had significantly lower rates of dental visits than did the moderately high risk group. Additionally, the rates of dental visits in the very high risk group had been declining annually. Contrastingly, the very high risk group had significantly higher rates of regular visits at medical institutions specializing in internal medicine than did the moderately high risk group over the entire period. Furthermore, we examined whether the rates of dental visits of CKD-diagnosed participants differs depending on the degree of impaired glucose tolerance (Fig. 3). The non-impaired glucose tolerance, HbA1c $>6.0$ or $\mathrm{FPG}>120 \mathrm{mg} / \mathrm{dL}$, and $\mathrm{HbAlc}>7.0$ or $\mathrm{FPG}>140$ $\mathrm{mg} / \mathrm{dL}$ groups were compared. In the group with moderately increased risk, the rates of dental visits decreased significantly as control over diabetes mellitus deteriorated (Fig. 3a). In the group with very high risk, there was no significant difference because of the small number of people, but the rate of dental visits tended to decrease as the control status over diabetes de-

Table 2. Classification of CKD-Diagnosed Participants That Attended Checkups in 2015

\begin{tabular}{llllll}
\hline GFR stage & eGFR $\left(\mathbf{m L} / \mathbf{m i n} / \mathbf{1 . 7 3 \mathbf { m } ^ { 2 } )}\right.$ & Proteinuria $(-)$ or $( \pm)$ & Proteinuria $(+)$ & Proteinuria $(++)$ or $(+++)$ & Unknown \\
\hline G1 (risk) & $\geq 90$ & 1,264 (green) & 46 (yellow) & 10 (orange) & 5 \\
G2 (risk) & $60-89$ & 8,597 (green) & 217 (yellow) & 52 (orange) & 9 \\
G3a (risk) & $45-59$ & 1,718 (yellow) & 85 (orange) & 38 (red) & 1 \\
G3b (risk) & $30-44$ & $170($ orange) & 24 (red) & 17 (red) & 0 \\
G4 (risk) & $15-29$ & 8 (red) & 9 (red) & 15 (red) & 0 \\
G5 (risk) & $<15$ & 0 (red) & 2 (red) & 2 (red) & $14($ dialysis) \\
\hline
\end{tabular}

Red: very high risk; orange: high risk; yellow: moderately increased risk; green: low risk or no CKD. 

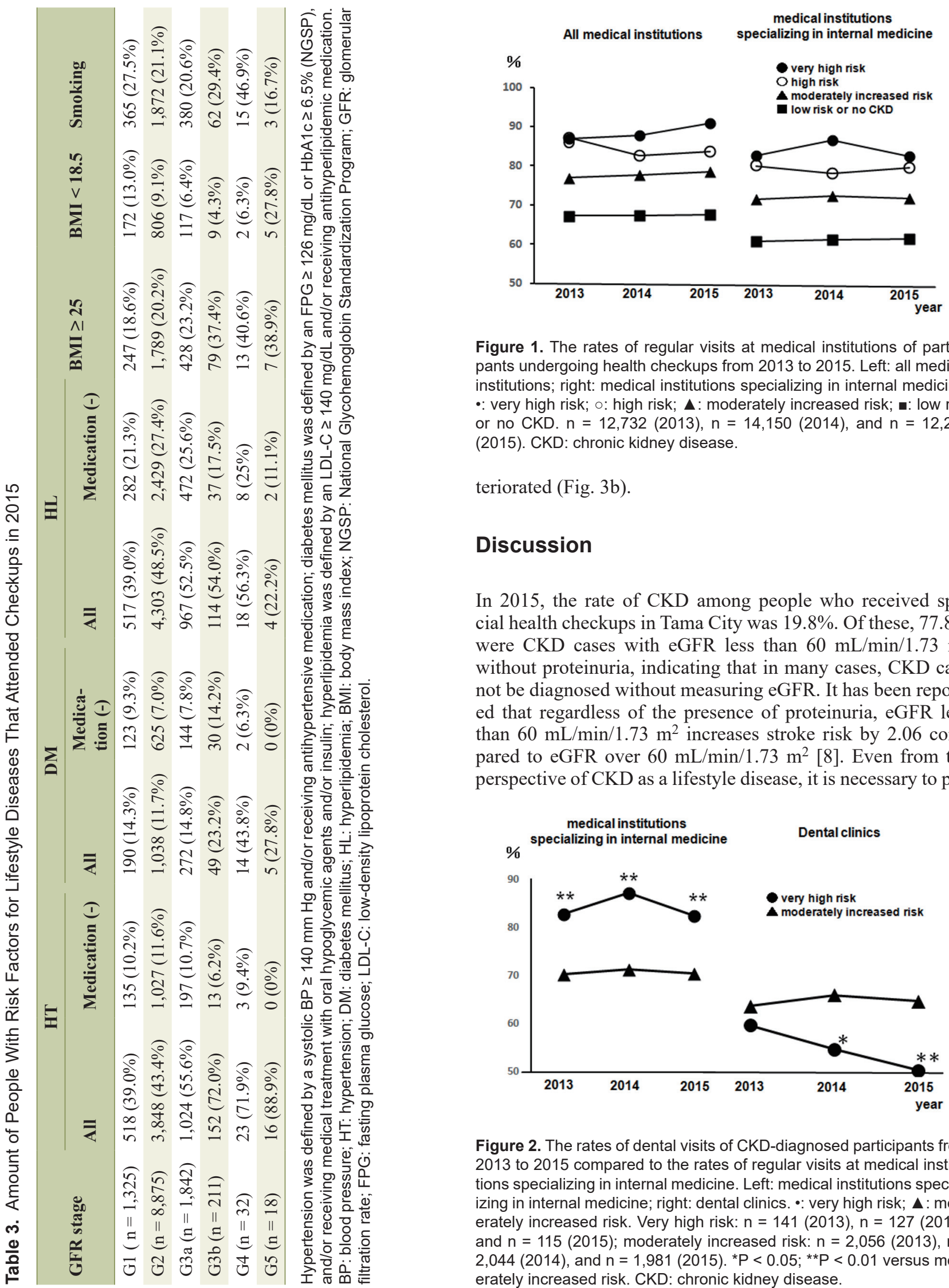

Figure 1. The rates of regular visits at medical institutions of participants undergoing health checkups from 2013 to 2015. Left: all medical institutions; right: medical institutions specializing in internal medicine. $\because$ very high risk; $\circ$ : high risk; $\boldsymbol{\Delta}$ : moderately increased risk; $\mathbf{m}$ : low risk or no CKD. $n=12,732$ (2013), $n=14,150$ (2014), and $n=12,274$ (2015). CKD: chronic kidney disease.

teriorated (Fig. 3b).

\section{Discussion}

In 2015, the rate of CKD among people who received special health checkups in Tama City was $19.8 \%$. Of these, $77.8 \%$ were CKD cases with eGFR less than $60 \mathrm{~mL} / \mathrm{min} / 1.73 \mathrm{~m}^{2}$ without proteinuria, indicating that in many cases, CKD cannot be diagnosed without measuring eGFR. It has been reported that regardless of the presence of proteinuria, eGFR less than $60 \mathrm{~mL} / \mathrm{min} / 1.73 \mathrm{~m}^{2}$ increases stroke risk by $2.06 \mathrm{com}$ pared to eGFR over $60 \mathrm{~mL} / \mathrm{min} / 1.73 \mathrm{~m}^{2}$ [8]. Even from the perspective of CKD as a lifestyle disease, it is necessary to pay

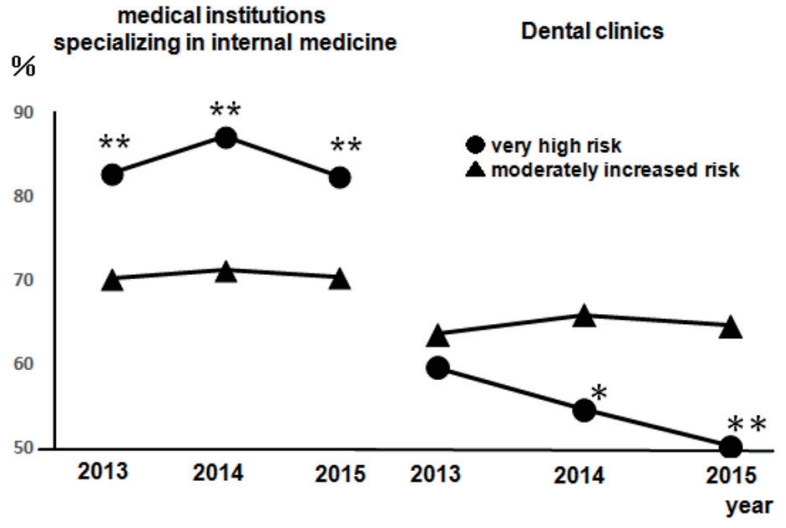

Figure 2. The rates of dental visits of CKD-diagnosed participants from 2013 to 2015 compared to the rates of regular visits at medical institutions specializing in internal medicine. Left: medical institutions specializing in internal medicine; right: dental clinics. $\bullet$ : very high risk; $\boldsymbol{\Lambda}$ : moderately increased risk. Very high risk: $n=141$ (2013), $n=127$ (2014), and $n=115$ (2015); moderately increased risk: $n=2,056$ (2013), $n=$ 2,044 (2014), and $n=1,981$ (2015). ${ }^{*} P<0.05$; ${ }^{* *} P<0.01$ versus moderately increased risk. CKD: chronic kidney disease. 


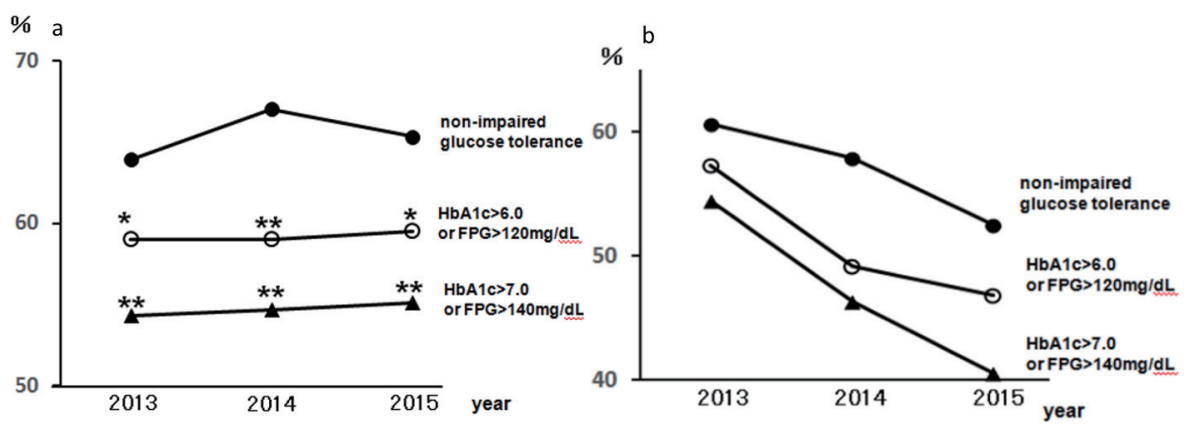

Figure 3. The rates of dental visits of CKD-diagnosed participants depending on the degree of impaired glucose tolerance from 2013 to 2015. (a) The rates of dental visits of CKD moderately increased risk group. $\because$ : Moderately increased risk with HbA1c $\leq 6.0$ and $\mathrm{FPG} \leq 120 \mathrm{mg} / \mathrm{dL}$ and not receiving medical treatment for diabetes: non-impaired glucose tolerance; $\circ$ : moderately increased risk with HbA1c $>6.0$ or FPG $>120 \mathrm{mg} / \mathrm{dL}$; $\boldsymbol{\Delta}$ : moderately increased risk with HbA1c $>7.0$ or FPG $>140 \mathrm{mg} / \mathrm{dL}$. ${ }^{*} \mathrm{P}$ $<0.05$; ${ }^{* *} P<0.01$ versus non-impaired glucose tolerance. (b) The rates of dental visits of CKD very high risk group. $\bullet:$ very high risk with $\mathrm{HbA} 1 \mathrm{c} \leq 6.0$ and $\mathrm{FPG} \leq 120 \mathrm{mg} / \mathrm{dL}$ and not receiving medical treatment for diabetes: non-impaired glucose tolerance; $\circ$ : very high risk with $\mathrm{HbA} 1 \mathrm{c}>6.0$ or $\mathrm{FPG}>120 \mathrm{mg} / \mathrm{dL} ; \boldsymbol{\Delta}$ : very high risk with $\mathrm{HbA} 1 \mathrm{c}>7.0$ or FPG $>140 \mathrm{mg} / \mathrm{dL}$. CKD: chronic kidney disease; HbA1c: hemoglobin A1c; FPG: fasting plasma glucose.

heed to it even in the minor stages. Moreover, even in patients with eGFR less than $45 \mathrm{~mL} / \mathrm{min} / 1.73 \mathrm{~m}^{2}$, proteinuria of (-) or $( \pm)$ represented $7.3 \%$ of the entire CKD population, indicating the possibility of overlooking relatively high-level CKD if serum creatinine is not measured during checkups. Compared with the results of special health checkups in 2008, the proportion of CKD without proteinuria has increased in our research, and this trend is expected to increase as the number of elderly people increases [9]. The serum creatinine test was added as an item to the detailed health checkup in 2018 , but has yet to become a compulsory item.

A range of measures are necessary to prevent the progression and worsening of CKD. As arteriosclerosis-induced CKD caused by diabetes mellitus, hypertension, dyslipidemia, and obesity has continued to increase in the recent years, it is important to supervise the improvement in lifestyle factors in the early stages of the disease $[2,10,11]$. Investigation of lifestyle disease comorbidity and smoking status revealed increases in hypertension as the CKD stages worsen. Even in CKD stage G3b and above, many had not received antihypertensive drugs despite having high BP, and treatment intervention is desirable from the viewpoint of suppression of cerebrocardiovascular events $[12,13]$. Diabetes mellitus, dyslipidemia, obesity, and smoking comorbidity rates are observed to increase up to CKD stage G4, and then decline from stage G5. As 14 of the 18 CKD stage G5 participants were undergoing dialysis in this study, this was speculated to be a contributing factor. As reported earlier, management of smoking and control of these factors have emphasized as potentially important in preventing the development of cerebrovascular disease and the development and progression of CKD [10, 14-16].

A large number of the very high risk group and high risk group regularly visited medical institutions. In both groups, however, there were not a few people who regularly visited medical institutions other than medical institutions specializing in internal medicine. It is thought that some of them were taking other treatments without knowing that the person suffers from CKD. In recent years, in some institutions in
Japan, "CKD sticker" has been placed on medicine record handbook carried by the patient to promote the distribution of renal function information. We started using multiple "CKD stickers" differentiated for each stage of CKD. If a drug is prescribed to a patient with renal dysfunction, the pharmacy also can check that the prescription is appropriate based on that information.

In contrast to regular visits at medical institutions specializing in internal medicine, the rate of dental visits declined as CKD risk increased. Results from a large-scale epidemiology survey of citizens indicated that even after adjusting for risk factors such as advanced age, smoking, hypertension, obesity and diabetes mellitus, periodontal disease patients had much higher rates of CKD than those with healthy gums $[17,18]$. Periodontal disease is a chronic inflammation that occurs from the body's immune response and periodontal bacteria in the periodontium. It is thought that worsening CKD could occur due to impaired vascular endothelial function brought about by inflammatory cytokines and reactive oxygen species produced by the periodontium or direct impairments from periodontal bacteria or bacteria producing organisms. It has been reported that treatment of periodontal disease for patients with CKD actually recovered kidney function [19] and improved inflammatory response [20]. Furthermore, difficulties in conducting dietary treatments such as reduced salt in the presence of severe periodontal disease are often encountered in clinical practice. Based on these results, we thought that failure to visit dentistry, that is, failure to treat periodontal disease, may contribute to the deterioration of CKD.

In this study, as diabetes mellitus management worsens in CKD-diagnosed participants, the rate of dental visits declined further. Periodontal disease has been reported to be a risk factor for renal function decline and mortality in patients with diabetes mellitus [21, 22]. Periodontal disease incidence rates have been reported to increase as glucose management worsens [23]. Periodontal disease is highly frequent in patients with diabetes mellitus, and as it deteriorates easily, it is thought to be related to basophil dysfunction, inhibition of collagen 
synthesis, periodontal ligament fibroblast dysfunction, microcirculation disorders, impaired immune function, and inflammatory tissue destruction of advanced glycation end products in diabetes mellitus [24]. A recent meta-analysis also reported a $0.4 \%$ reduction in $\mathrm{HbA} 1 \mathrm{c}$ due to treatment of periodontal disease in type 2 diabetes [25]. According to the results of Tama City specific health checkups, the rates of dental visits in the very high risk group had been declining each year, recommending that dental visits are also regarded as important in preventing the development and worsening of CKD and diabetic kidney disease.

The present study was constrained by several limitations. Firstly, the participants were limited by age and insurance coverage, as people with social insurance and/or aged $<40$ or $\geq$ 75 years at the onset were not eligible for these specific health checkups. Therefore, the overall prevalence and incidence of CKD were in all likelihood underestimated. Secondly, the diagnosis of CKD was based solely on the results of a single health checkup. CKD is defined as having aberrant test results over a period of over 3 months, and this too may have resulted in overestimating the number of CKD-diagnosed participants. Thirdly, uric acid was not measured in this study, even though it is a risk factor of CKD, as it was not compulsory to test it in the specific checkups. However, Tama City, recognizing its significance, added it as a compulsory test from the start of the 2016 financial year. As such, we were unable to add it to the current analysis, but this ought to be considered in future analyses to ensure better estimates.

\section{Conclusions}

In the current research we investigated the aggravating factors of CKD based on information from specific checkups and health insurance claims conducted between 2013 and 2015. In 2015, the authors established a new nephrology department at the Tama City University Hospital and cooperated with the TAMA CITY Medical Association to strengthen ties between primary care doctors, dentists, and pharmacists. We have also started a prospective study of whether periodontal disease treatment is effective in suppressing CKD progression. It is hoped that these results will play at least a minor role in preventing the progression and worsening of CKD.

\section{Acknowledgments}

We would like to thank Mr. Junichi Murata for his contribution to the statistical analyses.

\section{Financial Disclosure}

The TAMA MED project-CKD is supported by the TAMA CITY Medical Association. The sponsors had no role in the design and conduct of the study, in the collection, analysis and interpretation of data, in the preparation of the manuscript, or in the review or approval of the manuscript.

\section{Conflict of Interest}

EK received remuneration from Daiichi-Sankyo, Bristol-Myers Squibb, and Ono Pharmaceutical. Other authors have declared that no conflict of interest exists.

\section{Informed Consent}

Not applicable.

\section{Author Contributions}

TK performed research including data collection and analysis, and wrote the paper. HN, HS and YT performed research including data collection and participated discussion. EK, HF and ST designed research, study and provided expert clinical knowledge to revise critically.

\section{References}

1. National Kidney F. K/DOQI clinical practice guidelines for chronic kidney disease: evaluation, classification, and stratification. Am J Kidney Dis. 2002;39(2 Suppl 1):S1266.

2. Yamagata $\mathrm{K}$, Makino $\mathrm{H}$, Akizawa $\mathrm{T}$, Iseki $\mathrm{K}$, Itoh $\mathrm{S}$, Kimura K, Koya D, et al. Design and methods of a strategic outcome study for chronic kidney disease: frontier of renal outcome modifications in Japan. Clin Exp Nephrol. 2010;14(2):144-151.

3. Oda E, Watanabe K. Japanese criteria of metabolic syndrome. Circ J. 2006;70(3):364.

4. Kodani E, Kaneko T, Fujii H, Nakamura H, Sasabe H, Tamura Y, Shimizu W. Prevalence and incidence of atrial fibrillation in the general population based on national health insurance special health checkups- TAMA MED Project-AF. Circ J. 2019;83(3):524-531.

5. Fujii H, Kodani E, Kaneko T, Nakamura H, Sasabe H, Tamura Y. "Yubi-wakka" (Finger-Ring) test: a tool to detect prefrailty in elderly populations, a pilot study. J Clin Med Res. 2019;11(9):623-628.

6. Matsuo S, Imai E, Horio M, Yasuda Y, Tomita K, Nitta $\mathrm{K}$, Yamagata $\mathrm{K}$, et al. Revised equations for estimated GFR from serum creatinine in Japan. Am J Kidney Dis. 2009;53(6):982-992.

7. Kidney Disease: Improving Global Outcomes (KDIGO) CKD Work Group. KDIGO 2012 clinical practice guideline for the evaluation and management of chronic kidney disease. Kidney Int Suppl. 2013;3:1-150.

8. Ninomiya T, Kiyohara Y, Tokuda Y, Doi Y, Arima H, Harada A, Ohashi Y, et al. Impact of kidney disease and blood pressure on the development of cardiovascular disease: an overview from the Japan Arteriosclerosis Longitudinal Study. Circulation. 2008;118(25):26942701.

9. Iseki K, Asahi K, Moriyama T, Yamagata K, Tsuruya K, 
Yoshida H, Fujimoto S, et al. Risk factor profiles based on estimated glomerular filtration rate and dipstick proteinuria among participants of the Specific Health Check and Guidance System in Japan 2008. Clin Exp Nephrol. 2012;16(2):244-249.

10. Yamagata K, Ishida K, Sairenchi T, Takahashi H, Ohba S, Shiigai T, Narita M, et al. Risk factors for chronic kidney disease in a community-based population: a 10-year follow-up study. Kidney Int. 2007;71(2):159-166.

11. Wakasugi M, Kazama J, Narita I, Iseki K, Fujimoto S, Moriyama T, Yamagata K, et al. Association between overall lifestyle changes and the incidence of proteinuria: a population-based, cohort study. Intern Med. 2017;56(12):1475-1484.

12. Ninomiya T, Perkovic V, Gallagher M, Jardine M, Cass A, Arima H, Anderson C, et al. Lower blood pressure and risk of recurrent stroke in patients with chronic kidney disease: PROGRESS trial. Kidney Int. 2008;73(8):963970.

13. Ontarget Investigators, Yusuf S, Teo KK, Pogue J, Dyal L, Copland I, Schumacher H, et al. Telmisartan, ramipril, or both in patients at high risk for vascular events. N Engl J Med. 2008;358(15):1547-1559.

14. Manttari M, Tiula E, Alikoski T, Manninen V. Effects of hypertension and dyslipidemia on the decline in renal function. Hypertension. 1995;26(4):670-675.

15. Fox CS, Larson MG, Leip EP, Culleton B, Wilson PW, Levy D. Predictors of new-onset kidney disease in a community-based population. JAMA. 2004;291(7):844-850.

16. Yoshida T, Takei T, Shirota S, Tsukada M, Sugiura H, Itabashi M, Ogawa T, et al. Risk factors for progression in patients with early-stage chronic kidney disease in the Japanese population. Intern Med. 2008;47(21):18591864.
17. Fisher MA, Taylor GW, Shelton BJ, Jamerson KA, Rahman M, Ojo AO, Sehgal AR. Periodontal disease and other nontraditional risk factors for CKD. Am J Kidney Dis. 2008;51(1):45-52.

18. Kshirsagar AV, Moss KL, Elter JR, Beck JD, Offenbacher $\mathrm{S}$, Falk RJ. Periodontal disease is associated with renal insufficiency in the Atherosclerosis Risk In Communities (ARIC) study. Am J Kidney Dis. 2005;45(4):650-657.

19. Artese HP, Sousa CO, Luiz RR, Sansone C, Torres MC. Effect of non-surgical periodontal treatment on chronic kidney disease patients. Braz Oral Res. 2010;24(4):449454.

20. Vilela EM, Bastos JA, Fernandes N, Ferreira AP, Chaoubah A, Bastos MG. Treatment of chronic periodontitis decreases serum prohepcidin levels in patients with chronic kidney disease. Clinics (Sao Paulo). 2011;66(4):657-662.

21. Saremi A, Nelson RG, Tulloch-Reid M, Hanson RL, Sievers ML, Taylor GW, Shlossman M, et al. Periodontal disease and mortality in type 2 diabetes. Diabetes Care. 2005;28(1):27-32.

22. Shultis WA, Weil EJ, Looker HC, Curtis JM, Shlossman M, Genco RJ, Knowler WC, et al. Effect of periodontitis on overt nephropathy and end-stage renal disease in type 2 diabetes. Diabetes Care. 2007;30(2):306-311.

23. Tsai C, Hayes C, Taylor GW. Glycemic control of type 2 diabetes and severe periodontal disease in the US adult population. Community Dent Oral Epidemiol. 2002;30(3):182-192.

24. Loe H. Periodontal disease. The sixth complication of diabetes mellitus. Diabetes Care. 1993;16(1):329-334.

25. Teeuw WJ, Gerdes VE, Loos BG. Effect of periodontal treatment on glycemic control of diabetic patients: a systematic review and meta-analysis. Diabetes Care. 2010;33(2):421-427. 